\title{
La medicina basada en evidencia. Visión después de una década
}

\author{
Luz María Letelier $\mathbf{S}^{1,2^{*}}$, Philippa Moore ${ }^{3 *}$. \\ Evidence based medicine revisited
}

Evidence based medicine (EBM) appeared early in the 1990s and since then it has been developed and expanded worldwide. A decade later we summarize the history of EBM, the initial debates and the evolution to the current concept of evidence based health care (EBHC) as a tool for clinical decision making. We also describe the process of EBHC, some insights to current dilemmas and the situation of EBM in Chile (Rev Méd Chile 2003; 131: 939-46).

(Key Words: Clinical Medicine; Decission Support Systems, Clinical; Evidence based Medicine; Literature, modern)

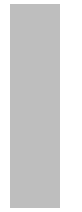

\footnotetext{
Recibido el 3 de junio, 2003. Aceptado el 19 de junio, 2003.

${ }_{1}^{1}$ Departamento de Medicina Interna, Pontificia Universidad Católica de Chile.

${ }^{2}$ Servicio de Medicina del Hospital Sótero del Río.

${ }^{3}$ Departamento de Medicina Familiar, Pontificia Universidad Católica de Chile.

*Coordinadoras de la Unidad de Medicina Basada en Evidencia, Universidad Católica de Chile. Santiago de Chile.
}

\section{Historia de la Medicina Basada en Evidencia}

En 1753 Lind demostró el beneficio de comer naranjas y limones para curar el escorbuto, en un estudio clínico de 12 pacientes ${ }^{1}$. La investigación clínica se desarrollaba lentamente, hasta dos siglos después en que apareció, en 1952, en el British Medical Journal, el primer ensayo clínico randomizado $^{2}$. Desde entonces la investigación clínica mantiene un crecimiento permanente, como describió el Dr. V. Valdivieso en esta revista ${ }^{3}$.

A fines de los 70 varios epidemiólogos clínicos, entre los que destacan D. Sackett, B. Haynes y P. Tugwell, se esforzaban por integrar la investigación clínica a la toma de decisiones para los pacientes. En

Correspondencia a: Dra. Luz María Letelier. Fax: 56-22897638. E mail: lmletel@med.puc.cl la década del 80 aparecieron en el Canadian Medical Association Journal las primeras publicaciones orientadas a revisar críticamente estudios publicados en revistas médicas. En 1990, G. Guyatt acuñó el término «Medicina Basada en Evidencia»(MBE) en un documento informal destinado a los residentes de Medicina Interna de la Universidad de Mc Master, en Canadá4. En 1992, el JAMA inició la serie de artículos «Users' Guides to the Medical Literature», iniciativa liderada por el Dr. Guyatt y epidemiólogos clínicos de universidades norteamericanas y europeas. Posteriormente, el término MBE fue ampliado para incluir otras especialidades médicas y no médicas que inciden en el cuidado de pacientes, denominándose «Evidence Based Health Care» (EBHC), que algunos traducen como «Cuidados de Salud Basados en Evidencia»o «Atención de Salud Basada en Evidencia», estas traducciones no han logrado aceptación general, por lo que seguiremos usando el término MBE, para referimos a la EBHC. 
La EBHC ha tenido impacto en sistemas de salud de países como Inglaterra y Canadá, que incorporaron los principios de la EBHC en sus políticas de salud y financian centros de estudios como el National Institute of Clinical Evidence (NICE) para desarrollar investigación en esta área.

\section{EVOLUCIÓN DE UNA POLÉMICA}

Como toda innovación que se precie de tal, la aparición y desarrollo de la MBE generó una fuerte polémica entre sus fanáticos defensores y su igualmente importante contraparte, ácidos detractores o defensores de la emedicina tradicional». Fue para los primeros un nuevo paradigma en la forma de hacer medicina y una filosofía de la práctica y la docencia clínica ${ }^{5,6}$. Ya no bastaba la experiencia sino que era obligatorio tener evidencia científica para tomar decisiones clínicas acertadas. Los detractores lo consideraron una exageración, la «deshumanización»de la medicina con mucha ciencia y poco arte ${ }^{7-10}$, el desconocimiento del valor de la experiencia y los expertos. ¿Acaso no es suficientemente evidente que repitiendo tratamientos que han sido útiles a otros enfermos, los próximos pacientes similares se beneficiarán de igual forma? La experiencia propia o de otros más antiguos o más expertos era base suficientemente sólida para la toma de decisiones $^{7,9}$. Estas dos posturas son, sin duda, extremas, pues si basamos nuestras decisiones sólo en evidencias científicas de alta calidad, la gran mayoría de nuestros pacientes quedaría sin tratamiento, ya que por diversas razones no existen evidencias científicas de alta calidad respecto a muchas de las decisiones clínicas que habitualmente tomamos. Por otro lado, si sólo basáramos nuestras decisiones médicas en experiencia previa quizás aún aplicaríamos sangrías a los pacientes con neumonía, como Louis en 1835¹, o lidocaína profiláctica a los pacientes con infarto al miocardio $^{11}$. El enorme volumen de la investigación clínica sería entonces un esfuerzo inútil.

\section{EXPERIENCIA VERSUS EVIDENCIA}

Parte de la polémica inicial se centró en antagonizar «experiencia» con «evidencia», en vez de definir qué llama evidencia la MBE. Tradicionalmente nos ha bastado como evidencia nuestra experiencia o la opinión de expertos, basada, a su vez, en su experiencia. La propuesta actual de la MBE es usar la mejor evidencia disponible para la toma de decisiones clínicas, sin desconocer la importancia de la experiencia. Este concepto de mejor evidencia implica necesariamente jerarquizar la evidencia, como se presenta en la Tabla 1 para estudios de terapia. La MBE propone que las revisiones sistemáticas (RS) de estudios clínicos randomizados (ECR) y los ECR son el mayor nivel de evidencia, no los únicos, sólo los de mayor jerarquía; es decir, basaremos nuestra decisión clínica en una RS o ECR si los hubiese, de lo contrario nos basaremos en los niveles siguien-

Tabla 1. Jerarquía de la evidencia

\begin{tabular}{|lll|}
\hline Jerarquía & Diseños & Sesgo \\
\hline I & Revisión sistemática y meta análisis & + \\
I & Estudios clínicos randomizados & + \\
II & Estudios observacionales: cohortes y caso-control & ++ \\
III & Reporte de series y casos clínicos & +++ \\
IV & Experiencia clínica & ++++ \\
\hline$+=$ mínimo sesgo & $++++=$ máximo sesgo
\end{tabular}


tes. Obviamente no podemos detener el proceso de toma de decisiones frente a un enfermo cuando no hay una RS o un ECR que avalen la decisión, pero sí debemos reconocer en qué tipo de evidencia basamos nuestra decisión.

La mayor jerarquía tiene relación inversa al posible sesgo de los distintos diseños de estudio (Tabla 1). La MBE llama validez interna» a la propiedad de los estudios que evalúa el riesgo de éstos de tener sesgo por su diseño. Muchos discrepan de esta jerarquía ${ }^{12}$, sin embargo, varios autores han demostrado diferencias significativas entre los resultados de estudios con mayor o menor validez interna ${ }^{13-15}$. El explosivo aumento de ECRs y RSs demuestra el interés de la comunidad científica por estos estudios.

Continuar antagonizando experiencia con evidencia retardará los procesos de cambio, pues ¿quién dudaría que la experiencia y la observación clínica son la mayor fuente de iniciativas para la investigación científica?

\section{CIENCIA VeRsUS ARTE}

Para los fanáticos de la MBE sólo la evidencia de alta jerarquía permitiría tomar decisiones adecuadas. Para sus detractores ello implicaba «deshumanizar» la medicina, la transformación de los clínicos en meros saplicadores de recetas de cocina», destruyendo así la relación médico-pa- ciente. Nuevamente estas dos posiciones extremas se han suavizado con el tiempo. La evidencia se considera siempre necesaria, pero nunca será suficiente para tomar decisiones. Hay múltiples factores a considerar al momento de tomar una decisión clínica, lo que nos lleva a la segunda propuesta de la MBE: el uso de la evidencia debe ser explícito y juicioso, vale decir que es importante cómo integramos la evidencia a nuestras decisiones. La aplicación indiscriminada de evidencia, aunque sea de alta jerarquía, sería en extremo peligrosa, pero la integración con buen criterio de la evidencia a otros factores determinantes en la toma de decisiones permitirán a nuestros pacientes beneficiarse de la mejor decisión.

El uso explícito y juicioso de la mejor evidencia disponible en la toma de decisiones frente a un paciente, no tiene por qué deteriorar la relación médico-paciente. Hacer este proceso explícito, informando al paciente y haciéndolo partícipe en las decisiones, crea una relación médico-paciente más transparente y participativa. No se debe confundir la toma de decisiones con la relación médico-paciente, ni las múltiples razones para la deshumanización de la medicina y el deterioro de la relación médico-paciente.

Así, se han ido reduciendo las diferencias que parecían irreconciliables ${ }^{16}$. Consideramos a la MBE un elemento más de juicio en el proceso de toma de decisiones clínicas (Figura 1), en que se

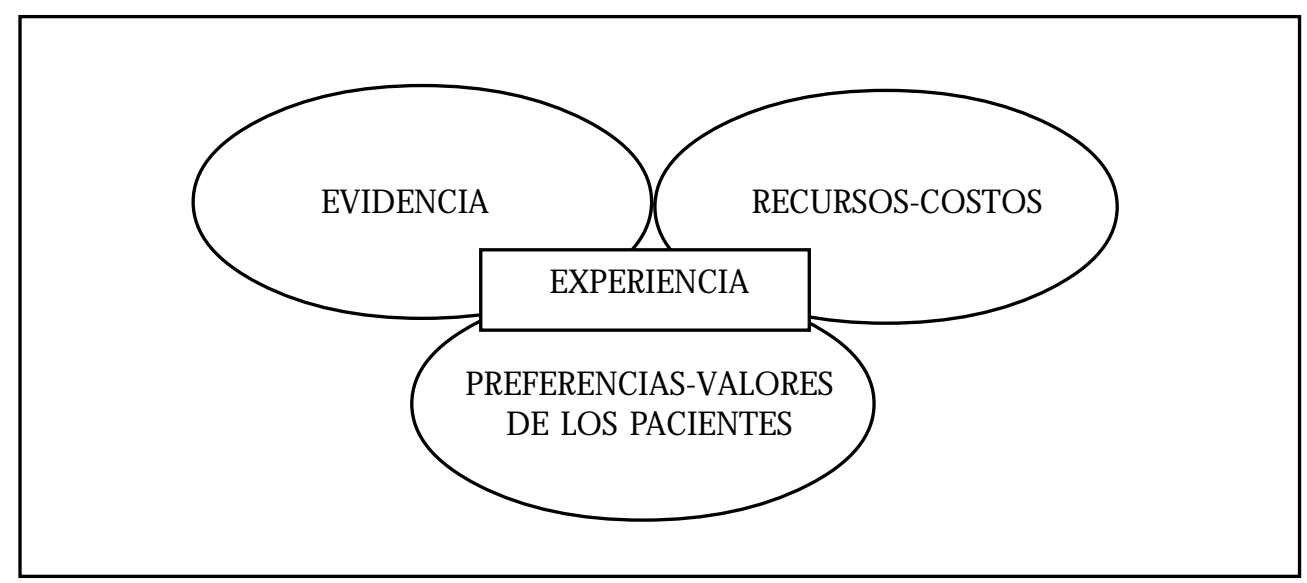

Figura 1. Toma de decisiones clínicas. 
conjugan tanto la experiencia y el juicio clínico, la realidad del entorno con los recursos disponibles, los valores o preferencias del paciente y la mejor evidencia proveniente de estudios clínicos. La MBE no pretende reinventar la rueda, sino hacer más explícito y sistemático un proceso que muchos clínicos hacen instintivamente. A quienes duden de la MBE como alternativa para mejorar la práctica clínica, les recomendamos leer las 7 alternativas a la $\mathrm{MBE}^{17}$.

\section{¿CómO PRACTICAR LA MBE?}

Practicar la MBE sólo tiene sentido en relación con una situación clínica que genere incertidumbre respecto a cuál es la mejor decisión clínica. Requiere de algunas habilidades (Figura 2), pero, en contra de lo que muchos piensan, no necesitamos ser expertos en bioestadística.

La incertidumbre la estructuramos en una pregunta clínica específica de 4 componentes fundamentales que incluyen el paciente, la intervención o tratamiento a evaluar, con su respectiva comparación y el outcome o evento observado o resultado final. Por ejemplo puedo retardar más el primer sangrado por várices esofágicas (outcome) si ligo las várices (la intervención) que si uso propanolol (comparación) en mi paciente con cirrosis y várices esofágicas que no ha tenido sangrados (el paciente)? Para respondernos, buscamos la evidencia pertinente usando estrategias de búsqueda eficiente en las bases de datos apropiadas. Luego hacemos el análisis crítico de la evidencia encontrada, para lo cual debemos conocer los fundamentos de los criterios que evalúan la validez interna y aplicabilidad de un estudio y saber interpretar los resultados en términos clínicamente relevantes, más allá de su significación. El análisis crítico constituye un pilar fundamental de la MBE, pues no todo lo publicado tiene la misma calidad científica, pero no se restringe a ello. Finalmente cerramos el proceso decidiendo cómo influirá esta nueva evidencia en la resolución de la incertidumbre inicial.

\section{MBE EN EL NUEVO MILENIO}

Con ayuda o no de la polémica inicial, lo cierto es que pocos quedaron indiferentes al concepto que más de una década después está ampliamente difundido.

El enorme desarrollo de la investigación científica hace que el volumen de información sea inmanejable para el clínico con escaso tiem-

Incertidumbre en la toma de decisiones

Estructurar una pregunta clínica específica

Realizar una búsqueda eficiente

\section{$\downarrow$}

Realizar el análisis crítico

$\rightarrow$ Validez interna

$\rightarrow$ Interpretación de resultados

$\rightarrow$ Aplicabilidad o validez externa

Resolver la incertidumbre

Figura 2. Proceso de la MBE. 
po para dedicar al estudio. Sólo en 20 revistas importantes de Medicina Interna se publican 6.000 artículos al año ${ }^{18}$ y la Cochrane Library registra 362.540 ECR (2003 issue 2). Paralelamente, el desarrollo de la computación permitió la creación de bases de datos electrónicas y la Internet su más libre y fácil acceso. En este contexto la MBE es una herramienta útil para mantenernos al día en la información acercando la investigación científica a la práctica clínica, como pretendían sus iniciadores.

Actualmente la MBE se desarrolla en tres niveles:

1. Quienes generan evidencia son aquellos que realizan investigación clínica, sea ésta primaria como reporte de casos, estudios observacionales y ECRs; o la llamada investigación secundaria como son las RSs o las guías de práctica clínica (GPC). En todas ellas la MBE contribuye a mejorar la calidad de estos estudios.

2. Quienes practican la MBE son aquellos que realizan el proceso completo de la MBE. Esta práctica permite al clínico resolver las incertidumbres de su quehacer diario, las que pueden ser posteriormente aplicadas a otras situaciones similares. También el proceso puede ser resumido y difundido para ser utilizado por otros.

3. Quienes usan la MBE son aquellos que, estando de acuerdo con utilizar la MBE para tomar decisiones, no tienen las herramientas o el tiempo suficiente para realizar el proceso completo, utilizan en cambio evidencia ya filtrada y analizada por quienes practican la MBE.

Han nacido así muchas revistas, bases de datos y sitios de la Internet que llamaremos globalmente «Recursos MBE».

Los Centros de Investigación Secundaria generan evidencia llamada investigación secundaria como las RS, GPC y estudios de costo-efectividad. Uno de ellos, la Cochrane Collaboration (CC) (www.cochrane.org), es una organización sin fines de lucro cuya historia y fundamentos fue descrita en esta revista ${ }^{3}$, tiene como principal propósito generar RS de terapias. Su desarrollo se manifiesta en el número creciente de RSs, que de aproximadamente 800 títulos en $1997^{3}$ registra actualmente 2.935. El National Health Service Centre for Research \& Development crea RS y estudios de costo-efectividad. Otra fuente de investigación secundaria son las organizaciones dedicadas a la creación de GPC como la National Clearinghouse en los EE.UU. (www.guideline.gov) y New Zealand Guidelines (www.nzgg.org.nz/library.cfm).

Los Centros de Publicaciones Secundarias filtran, analizan y sintetizan en formatos amigables estudios publicados en revistas tradicionales. El ACP Journal (www.acpjc.org) y Clinical Evidence (www.clinicalevidence.com) están entre los pioneros y más utilizados en Medicina Interna. La CC también revisa y sintetiza RSs realizadas por otros grupos. Actualmente existen 25 revistas secundarias, 11 secciones de resúmenes en otras revistas, 13 〈journal clubs»en línea y 12 〈Cat banks» ${ }^{19}$. Los CAT (Critically Appraised Topic) creados por Sackett y su grupo dieron origen a múltiples derivados como los POEM, BestBETS, y nuestro producto nacional el ECLPSE (Enfoque Clínico de Problemas Sustentados en Evidencia) (www.escuela.med.puc.cl/paginas/Recursos/ MBE2001/).

Recursos de Búsqueda: A partir de la MBE surgen recursos que simplifican y hacen más eficiente la búsqueda. Los meta-buscadores son interfases que buscan en muchas bases de datos simultáneamente. Tripdatabase, por ejemplo, se creó en 1997 en un intento por reunir todos los Recursos MBE disponibles en Internet y actualmente revisa 75 sitios. PubMed, el buscador gratuito de MEDLINE, cuenta con el «clinical query», programa eficiente que selecciona estudios de alta jerarquía.

Así, el desarrollo de la MBE ha facilitado el acceso y reducido el tiempo necesario para que quienes no disponen de mucho tiempo puedan llegar a la mejor evidencia, manteniéndose al día y brindando una mejor atención a sus pacientes.

\section{MBE EN CHILE}

Buscamos cuánto se publica sobre MBE en revistas chilenas indizadas en MEDLINE: en PubMed 
encontramos 11.375 publicaciones pertinentes que disminuyeron a 163 al limitarlas al español, y de éstas sólo 4 estaban publicadas en la Revista Médica de Chile $3,9,10,20$. En ellas se desarrolló la misma polémica internacional, pero no encontramos publicaciones posteriores al año 2000.

Existen varios grupos en Chile que trabajan en distintos aspectos de la MBE. La Universidad de la Frontera creó hace 9 años el Centro de Capacitación, Investigación y Gestión para la Salud Basada en Evidencia, CIGES (www.ciges.cl) siendo representantes en Chile de la Red Mundial de Epidemiología Clínica (INCLEN). La Universidad de Chile tiene diversos grupos, destacando su Centro de Epidemiología Clínica y el Departamento de Ciencias Neurológicas. Este último firmó en 1994 un convenio de colaboración con la Universidad de Liverpool, dirigido a facilitar la apreciación crítica y síntesis de evidencia. En la Pontificia Universidad Católica de Chile, en Santiago, tenemos la Unidad de Medicina Basada en Evidencia, UMBE-UC $^{21}$. La Clínica Alemana de Santiago y otras instituciones privadas de salud también han hecho actividades de MBE. El mayor esfuerzo de estos grupos se ha canalizado en enseñar 0 difundir conceptos de MBE. Las RSs hechas por chilenos, han sido publicadas en Cochrane ${ }^{22-26}$ o en revistas extranjeras ${ }^{27,28}$, pero lamentablemente no han tenido gran difusión nacional.

El Ministerio de Salud creó en 1997 una Unidad de Medicina Basada en Evidencias, para evaluar la evidencia que respaldaba las intervenciones propuestas para ser incorporadas a la lista de prestaciones financiadas por FONASA. En 1999 se integró con la Unidad de Evaluación de Tecnologías Sanitarias, que fue la primera Agencia de Evaluación de Tecnologías Sanitarias del tercer mundo aceptada como miembro de INAHTA (International Network of Agencies of Health Technology Assessment). También estableció un convenio de colaboración mutua con la CC (Centro Iberoamericano). Desde 2003, la Librería Cochrane en castellano está disponible gratuitamente a través de la Biblioteca Virtual de Salud de la OPS (www.cochrane.bireme.br).

La comunidad médica chilena está motivada por la MBE, particularmente algunas sociedades científicas interesadas en crear GPC locales, solicitan apoyo de grupos de MBE para su correcto desarrollo metodológico, otras solicitan talleres o charlas de MBE como una actividad más de sus congresos anuales, o para cursos especiales.

Posiblemente parte del trabajo de los grupos de MBE esté en la Internet, en publicaciones extranjeras 0 en revistas nacionales no indizadas en MEDLINE. Nuestra propuesta es difundir estas actividades y trabajar en conjunto para enriquecer el trabajo de todos. Esperamos que la nueva Sección creada en esta Revista contribuya a este objetivo.

\section{Desafíos DE la MBE}

Establecimos el rol de la MBE en la práctica clínica del médico individual, pero existe también un rol para la MBE en las grandes decisiones de las políticas de salud, como ocurre en Canadá y en el Reino Unido. Las GPC basadas en evidencias de buena calidad creadas, difundidas e implementadas por los organismos de salud es uno de los campos en desarrollo de la MBE.

A pesar de los avances, el acceso a la información no es uniforme, particularmente en países en desarrollo y de lengua no inglesa, como el nuestro. La excesiva carga de trabajo y la falta de tiempo son males de esta época y no podemos negar que el proceso de la MBE además de habilidades necesita tiempo. El consumo de tiempo y energía aumenta además por la inevitable necesidad de actualizarse en inglés. Independiente del lugar de origen, muchos autores publican en inglés y los recursos de la MBE están en su mayoría en inglés. Un entrenamiento apropiado y el desarrollo de nuevos recursos MBE, idealmente en español, disminuirá considerablemente el tiempo requerido para practicar o usar la MBE en Chile. Aunque la Internet facilita el acceso a la información, también es un arma de doble filo si se utiliza sin mediar el análisis crítico de la información presentada, particularmente cuando la utilizan personas que desconocen el método científico.

¿Uso o abuso del término sevidencia»? No recordamos haber asistido en los últimos años a una exposición científica sin escuchar ła evidencia muestra......», o algo similar. Incluso nuestros alumnos defienden sus respuestas aludiendo a la evidencia; sin embargo, muy pocos van más allá y realmente describen la jerarquía de la evidencia aludida. Muchos piensan que basta leer la conclusión del abstract de un artículo publicado en una 
revista de renombre, para estar basado en evidencia. Esto indudablemente constituye un grave riesgo de desvirtuar la real intención de la MBE.

Finalmente, sabemos que conocer no es lo mismo que implementar. Muchos saben que fumar es perjudicial, pero el sólo conocimiento no cambia los hábitos. Así mismo, el conocer nueva evidencia no asegura su implementación cuando así corresponde. En el siglo XVIII, la Armada Británica tardó medio siglo en incluir cítricos en las raciones de las tripulaciones después del estudio de Lind ${ }^{1}$, en los 80 la recomendación de emplear trombolisis en el infarto al miocardio se difundió más de una década después de estar demostrado su beneficio ${ }^{11}$.

\section{REFERENCIAS}

1. Pocock SJ. The historical development of clinical trials. Chapter 2, pg 14-15. In Clinical Trials, John Wiley \& Sons, Toronto 1984.

2. Daniels M, HiL AB. Chemotherapy of pulmonary tuberculosis in young adults. BMJ 1952; I: 1162-8.

3. Valdivieso V. La Medicina Basada en Evidencias. Rev Méd Chile 1997; 125: 1103-9.

4. GuyatT G. Users' Guides to the Medical Literature. A manual for Evidence-Based Clinical Practice. Ed G Guyatt, D Rennie, 2002, pg xiv.

5. Evidence Based Medicine Working Group. Evidence Based Medicine. A new approach to teaching the practice of medicine. JAMA 1992; 268: 2420-5.

6. Rosenberg W, Donald A. Evidence based medicine: an approach to clinical problem solving. BMJ 1995; 310: 1122-6.

7. Smith BH, Taylor RJ. Medicine, a healing or a dying art? Br J Gen Pract 1996; 46: 249-51.

8. Glass RM. The Patient-Physician relationship. JAMA 1996; 275: 147-8.

9. Duclos J. Medicina basada en evidencias: ¿una estrategia que acerca o aleja de la medicina interna? Rev Méd Chile 1999; 127: 1398-402.

10. Cantu P. Medicina basada en evidencias. Rev Méd Chile 2000; 128: 553.

\section{CONCLUSIÓN}

Aunque quedan interrogantes como: ¿cuánto de la MBE conocemos y practicamos los clínicos?, ¿qué tipo de evidencia usamos en nuestra toma de decisiones?, ¿cuánto demoramos en integrar la evidencia a nuestra toma de decisiones? o ¿cómo integramos la evidencia a la toma de decisiones? Después de una década de haberse definido la MBE quisimos resumir su evolución y situación actual, dejando planteadas estas interrogantes e invitando a la comunidad chilena dedicada a MBE a trabajar conjuntamente.

11. Antman EM, Lau J, Kuperunk B, Mosteler F, CHALMERS T. A comparison of results of meta analyses of randomized controlled trials and recommendations of clinical experts: treatments for myocardial infarction. JAMA 1992; 268: 240-8.

12. Feinstein AR, Horwitz RI. Problems in the sevidence» of evidence based medicine». Am J Med 1997; 103: 529-35.

13. Chalmers TC, Celano P, Sacks HS, Smith H Jr. Bias in treatment assignment in controlled clinical trials. N Engl J Med 1983; 309: 1358-6.

14. Schulz KF, Chalmers I, Hayes RJ, Altman DG. Empirical evidence of bias: dimensions of methodological quality associated with estimates of treatment effects in controlled trials. JAMA 1995; 273: 408-12.

15. Moher D, JoNes A, Cook DJ, JADAD AR, Moher M, TugweLl P ET AL. Does quality of reports of randomized trials affect estimates of intervention efficacy reported in meta analyses? Lancet 1998; 352: 609-13.

16. Greenhalgh T, Worral J. From EBM to CMS: the evolution of context sensitive medicine. J Evaluation Clin Practice 1997; 3: 105-8.

17. IsAacs D, FitZgerald D. Seven alternatives to evidence based medicine. BMJ 1999; 319: 1618. 
18. Davidoff F, Haynes B, SACkett HD, Smith R. Evidence based medicine. A new journal to help doctors identify the information they need. BM 1995; 310: 1085-6.

19. www.infodoctor.org/rafabravo/revistassecun.htm\#rs

20. Montero J. Medicina basada en evidencias. Rev Méd Chile 2000; 128: 553-4.

21. www .escuela.med.puc.cl/paginas/Recursos/ MBE2001

22. Fellowes D, Wilkinson S, Moore P. Communication skills training for health care professionals working with cancer patients, their families and/or carers (Cochrane Review). In: The Cochrane Library, Issue 2, 2003. Oxford.

23. Verdugo RJ, Salinas RS, Castilo J, Cea JG. Surgical versus non surgical treatment for carpal tunnel syndrome (Cochrane Review). In: The Cochrane Library, Issue 2, 2003. Oxford.

24. Counsell C, Salinas R, Warlow C, Naylor R. Patch angioplasty versus primary closure for carotid endarterectomy (Cochrane Review). In: The Cochrane Library, Issue 2, 2003. Oxford.

25. Rojas MP, Telaro E, Russo A, Fossati R, Paui D, Rossew Del Turco M et al. Follow up strategies for women treated for early breast cancer (Cochrane Review). In: The Cochrane Library, Issue 2, 2003. Oxford.

26. Salinas RA, Alvarez G, Alvarez MI, Ferreira J. Corticosteroids for Bell's palsy (idiopathic facial paralysis) (Cochrane Review). In: The Cochrane Library, Issue 2, 2003. Oxford.

27. Salinas R, Counsell C, Prasad K, Gelband H, Garner P. Ministry of Health, Santiago, Chile. Treating neurocysticercosis medically: a systematic review of randomized, controlled trials. Trop Med Int Health 1999; 4: 713-8.

28. Letelier LM, Udol K, Ena J, Weaver B, Guyatt G. Effectiveness of amiodarone for conversion of atrial fibrillation to sinus rhythm. A meta analysis. Arch Intern Med 2003; 163: 777-85.

\section{Agradecimientos}

Al Dr. Vicente Valdivieso, por la revisión de este manuscrito y a los Drs. Rodrigo Salinas, Jorge Sapunar y Gabriel Cea por aportarnos datos de sus Centros. 